\title{
Quantization coefficients in infinite systems
}

\author{
Eugen Mihailescu and Mrinal Kanti Roychowdhury
}

\begin{abstract}
We investigate quantization coefficients for probability measures $\mu$ on limit sets, which are generated by systems $\mathcal{S}$ of infinitely many contractive similarities and by probabilistic vectors. The theory of quantization coefficients for infinite systems has significant differences from the finite case. One of these differences is the lack of finite maximal antichains, and another is the fact that the set of contraction ratios has zero infimum; another difference resides in the specific geometry of $\mathcal{S}$ and of its noncompact limit set $J$. We prove that, for each $r \in(0, \infty)$, there exists a unique positive number $\kappa_{r}$, so that for any $\kappa<\kappa_{r}<\kappa^{\prime}$, the $\kappa$-dimensional lower quantization coefficient of order $r$ for $\mu$ is positive, and we give estimates for the $\kappa^{\prime}$-upper quantization coefficient of order $r$ for $\mu$. In particular, it follows that the quantization dimension of order $r$ of $\mu$ exists, and it is equal to $\kappa_{r}$. The above results allow one to estimate the asymptotic errors of approximating the measure $\mu$ in the $L_{r}$-Kantorovich-Wasserstein metric, with discrete measures supported on finitely many points.
\end{abstract}

\section{Introduction and general setting}

The theory of quantization studies the process of approximating probability measures, which are invariant for certain systems, with discrete probabilities having a finite number of points in their support. Of particular interest are the types of behaviors which may be encountered in this quantization process for various measures.

Let us consider, in general, a probability measure $\mu$ on $\mathbb{R}^{d}$, a number $r \in$ $(0, \infty)$, and a natural number $n \in \mathbb{N}$. Then, the $n$th quantization error of order $r$ of $\mu$ is defined by

$$
V_{n, r}(\mu):=\inf \left\{\int d(x, \alpha)^{r} d \mu(x): \alpha \subset \mathbb{R}^{d}, \operatorname{card}(\alpha) \leq n\right\},
$$

where $d(x, \alpha)$ denotes the distance from an arbitrary point $x$ to the set $\alpha$ with respect to the Euclidean norm on $\mathbb{R}^{d}$. If $\int\|x\|^{r} d \mu(x)<\infty$, then there exists some set $\alpha$ for which the infimum is achieved (see [GL1]). A set $\alpha$ for which the infimum is achieved is called an optimal set of $n$-means or an $n$-optimal set of order $r$, for the probability $\mu$ and for $0<r<\infty$.

For $s>0$, the $s$-dimensional upper and lower quantization coefficients of order $r$ for the probability measure $\mu$ are defined (see [GL1]), respectively, as

Kyoto Journal of Mathematics, Vol. 55, No. 4 (2015), 857-873

DOI $10.1215 / 21562261-3089118$, (C) 2015 by Kyoto University

Received January 27, 2014. Accepted August 15, 2014.

2010 Mathematics Subject Classification: 28A32, 28A80, 28A25, 60 B05.

Mihailescu's work partially supported by CNCSIS grant 2011-3-0269. 


$$
\begin{aligned}
& \overline{\mathcal{Q C}}_{r, s}(\mu):=\limsup _{n} n V_{n, r}(\mu)^{s / r} \quad \text { and } \\
& \underline{\mathcal{Q C}}_{r, s}(\mu):=\liminf _{n} n V_{n, r}(\mu)^{s / r} .
\end{aligned}
$$

We will be interested below in quantization coefficients for self-similar probability measures $\mu$ for infinite systems of contractive similarities $\mathcal{S}=\left(S_{1}, S_{2}, \ldots\right)$ and for infinite probability vectors $p=\left(p_{1}, p_{2}, \ldots\right)$. In this case, the theory and the techniques of the proof from the finite case do not work. In particular, we do not have finite maximal antichains, and also the set of the contraction ratios for the maps $S_{i}, i \geq 1$, has zero infimum.

Recall that, in the finite case, a finite self-similar system is determined by a set of contractive similarity mappings on $\mathbb{R}^{d}$, namely, $\left\{S_{1}, S_{2}, \ldots, S_{N}\right\}$ with contraction rates $s_{1}, s_{2}, \ldots, s_{N}$, for $N \geq 2$. By [H] for any probability vector $\left(p_{1}, p_{2}, \ldots, p_{N}\right)$ there exists a unique Borel probability measure $\mu$, known as a self-similar measure, and a unique nonempty compact fractal subset $J$ of $\mathbb{R}^{d}$, which is the support of $\mu$, satisfying the self-similarity conditions:

$$
\mu=\sum_{j=1}^{N} p_{j} \mu \circ S_{j}^{-1} \quad \text { and } \quad J=\bigcup_{j=1}^{N} S_{j}(J) .
$$

The finite iterated system $\left\{S_{1}, S_{2}, \ldots, S_{N}\right\}$ satisfies the open set condition if there exists a bounded nonempty open set $U \subset \mathbb{R}^{d}$ such that $\bigcup_{j=1}^{N} S_{j}(U) \subset U$ and $S_{i}(U) \cap S_{j}(U)=\emptyset$ for $1 \leq i \neq j \leq N$. The iterated system is said to satisfy the strong open set condition if there is an open set $U$ as above, so that $U \cap J \neq \emptyset$, where $J$ is the limit set of the system (see, e.g., [H], [F]).

The upper and lower quantization dimensions of order $r$ of $\mu$ are defined, respectively, as

$$
\bar{D}_{r}(\mu):=\limsup _{n \rightarrow \infty} \frac{r \log n}{-\log V_{n, r}(\mu)}, \quad \underline{D}_{r}(\mu):=\liminf _{n \rightarrow \infty} \frac{r \log n}{-\log V_{n, r}(\mu)} .
$$

If $\bar{D}_{r}(\mu)$ and $\underline{D}_{r}(\mu)$ coincide, then we call the common value the quantization dimension of order $r$ of the probability measure $\mu$, and it is denoted by $D_{r}(\mu)$. Quantization processes form a rich and far-reaching mathematical concept, with many applications (see, e.g., [GG], [GL1], [Za]).

Under the open set condition, Graf and Luschgy (see [GL1], [GL3]) showed that the quantization dimension $D_{r}(\mu)$ of order $r$ of the probability measure $\mu$ exists and satisfies the relation $\sum_{j=1}^{N}\left(p_{j} s_{j}^{r}\right)^{\frac{D_{r}}{r+D_{r}}}=1$. In fact they proved more, namely, that the quantization dimension $D_{r}(\mu)$ also satisfies the following growth conditions for quantization errors (see [GL2]):

$$
0<\liminf _{n} n V_{n, r}(\mu)^{\frac{D_{r}}{r}} \leq \limsup _{n} n V_{n, r}(\mu)^{\frac{D_{r}}{r}}<\infty .
$$

Under the open set condition, Lindsay and Mauldin (see [LM]) determined the quantization dimension of an $F$-conformal measure $m$ associated with a conformal iterated function system (IFS) determined by finitely many conformal mappings. They established a relationship between the quantization dimension 
and the temperature function of the thermodynamic formalism arising in multifractal analysis, and proved that the upper quantization coefficient of $m$ is finite; however, they left it open as to whether the lower quantization coefficient is positive. Using a class of finite maximal antichains, Zhu [Z] gave an answer. Later, following the same techniques as Lindsay and Mauldin and using Hölder's inequality, Roychowdhury [R1] gave a different proof of this fact.

In this paper, we are interested in the different case of infinite systems of similarities $\left(S_{n}\right)_{n \geq 1}$ with similarity ratios $\left(s_{n}\right)_{n \geq 1}$, respectively, satisfying the strongly separated condition. This setting presents several challenges, different from the finite case. For example in the infinite case, the fractal limit set $J$ of the system is not necessarily compact, in contrast to that of the finite case. The Hausdorff dimension of the limit set $J$ of an infinite conformal IFS is given in general only as the infimum of the values which make the pressure negative; there may be no zero of that pressure, unlike in the finite case. There are examples of infinite systems where the lower box dimension $\underline{\operatorname{dim}}(J)$ is strictly larger than $H D(J)=h$ and examples where the Hausdorff measure $H_{h}(J)$ is zero while for others $H_{h}(J)>0$ (see $\left.[\mathrm{MaU}]\right)$.

Also the boundary at infinity consisting of accumulation points of sequences of type $\left(S_{i}\left(x_{i}\right)\right)_{i}$ with distinct $i$ 's plays a role in the geometric properties of the system (see Mauldin and Urbański [MaU], Mihailescu and Urbański [MU1]). For example, Mihailescu and Urbański [MU1] studied the effects of overlaps and of the boundary at infinity on the dimensions of limit sets of infinite conformal IFSs.

For the case of invariant measures for finite or infinite IFS, for which the open set condition is not necessarily satisfied and there may exist overlaps, see Mihailescu and Urbański [MU1], [MU], [MU2].

Moreover, pertaining to our problem of quantization processes, we do not have finite maximal antichains, and the infimum of the contraction rates is zero, which makes the proofs from the finite case not work in the infinite situation.

As it turns out, estimating quantization coefficients in the infinite case is also very different from doing so in the finite case. By its intrinsic nature, quantization is a procedure of "fitting" a finite set in the noncompact fractal limit set $J$ in such a way that we obtain as much information as possible about the selfsimilar measure $\mu$ which is supported on $\bar{J}$. However, when dealing with an infinite system, usually no finite set $F$ can be placed properly such that every set $S_{j}(X), j \geq 1$, contains a point from $F$. This makes quantization for infinite systems very different from that for finite systems.

Let $\mu$ be the self-similar probability generated by the system $\left(S_{n}\right)_{n \geq 1}$ and by the probability vector $\left(p_{n}\right)_{n \geq 1}$ (see, e.g., [M]). The measure $\mu$ satisfies the following recursive formula:

$$
\mu=\sum_{j=1}^{\infty} p_{j} \mu \circ S_{j}^{-1} .
$$


The measure $\mu$ is supported on the compact closure $\bar{J}$ of the associated limit set $J$. (The precise definition will be given below in the General setting.)

We will prove in Theorem 2.1 that, under the strongly separated condition, for each $r \in(0, \infty)$ there exists a unique $\kappa_{r} \in(0, \infty)$ so that $\sum_{j=1}^{\infty}\left(p_{j} s_{j}^{r}\right)^{\frac{\kappa_{r}}{r+\kappa_{r}}}=1$, and for any $\kappa<\kappa_{r}$, the $\kappa$-dimensional lower quantization coefficient of order $r$ for the measure $\mu$ satisfies the following asymptotic condition:

$$
0<\liminf _{n \rightarrow \infty} n V_{n, r}(\mu)^{\frac{\kappa}{r}} \leq \limsup _{n \rightarrow \infty} n V_{n, r}(\mu)^{\frac{\kappa}{r}} .
$$

We also show in Theorem 2.1 that, for any $\kappa^{\prime}>\kappa_{r}$, the $\kappa^{\prime}$-dimensional upper quantization coefficient for $\mu$ is finite,

$$
\limsup _{n \rightarrow \infty} n V_{n, r}(\mu)^{\kappa^{\prime} / r}=0 .
$$

In particular, in Corollary 2.2 we prove that the quantization dimension of order $r$ of $\mu$ exists, and it is equal to $\kappa_{r}$ setting. As a consequence of the main results, we will prove in Corollary 2.5 a result about the asymptotic behavior in $n$ of the approximations in the $L_{r}$-Kantorovich-Wasserstein metric of the self-similar probability measure $\mu$, by discrete probability measures $Q$ which are supported on $n$ points.

We also give examples of self-similar measures for infinite systems for which we can obtain estimates on the quantization coefficients.

Some partial attempt to quantization in infinite IFS was tried by Roychowdhury [R2]. However, the proof of the main result in that paper is incorrect (see Remark 2.3 below); therefore, it was not used in the current paper. The ideas and methods in our current paper are completely different.

General setting. The $n$th quantization error for the probability $\mu$ gives, in essence, the minimal average distance (average with respect to $\mu$ ), from points in the support of $\mu$ to finite sets of cardinality $n$, and is defined (see [GL1]) by the formula:

$$
V_{n, r}(\mu):=\inf \left\{\int d(x, \alpha)^{r} d \mu(x): \alpha \subset \mathbb{R}^{d}, \operatorname{card}(\alpha) \leq n\right\} .
$$

Denote $e_{n, r}(\mu):=V_{n, r}(\mu)^{\frac{1}{r}}$. A set $\alpha \subset \mathbb{R}^{d}$ with $\operatorname{card}(\alpha) \leq n$ is called an $n$-optimal set of centers for $\mu$ of order $r$ or a $V_{n, r}(\mu)$-optimal set whenever we have

$$
V_{n, r}(\mu)=\int d(x, \alpha)^{r} d \mu(x)
$$

Let $X$ be a nonempty compact subset of $\mathbb{R}^{d}$ with $X=\operatorname{cl}(\operatorname{int} X)$. Let $\left(S_{j}\right)_{j=1}^{\infty}$ be an infinite set of contractive similarity mappings on $X$ whose contraction ratios are, respectively, $\left(s_{j}\right)_{j=1}^{\infty}$, that is, $d\left(S_{j}(x), S_{j}(y)\right)=s_{j} d(x, y)$ for all $x, y \in$ $X, 0<s_{j}<1, j \geq 1$. We shall assume in the rest of the paper that

$$
s:=\sup _{j \geq 1} s_{j}<1 .
$$

A word with $n$ letters in $\mathbb{N}=\{0,1,2, \ldots\}, \omega:=\omega_{1} \omega_{2} \cdots \omega_{n} \in \mathbb{N}^{n}$, is said to have length $n$, for $n \geq 1$. Define also $\mathbb{N}^{\text {fin }}:=\bigcup_{n \geq 1} \mathbb{N}^{n}$ to be the set of finite words of any length with letters in $\mathbb{N}$. For $\omega=\omega_{1} \omega_{2} \cdots \omega_{n} \in \mathbb{N}^{n}$, define 


$$
S_{\omega}=S_{\omega_{1}} \circ S_{\omega_{2}} \circ \cdots \circ S_{\omega_{n}} \quad \text { and } \quad s_{\omega}=s_{\omega_{1}} s_{\omega_{2}} \cdots s_{\omega_{n}} .
$$

The empty word $\emptyset$ is the only word of length 0 and $S_{\emptyset}=\operatorname{Id}_{X}$. For $\omega \in \mathbb{N}^{\text {fin }} \cup \mathbb{N}^{\infty}$ and for a positive integer $n$ smaller than the length of $\omega$, we denote by $\left.\omega\right|_{n}$ the word $\omega_{1} \omega_{2} \cdots \omega_{n}$. Notice that, given $\omega \in \mathbb{N}^{\infty}$, the compact sets $S_{\left.\omega\right|_{n}}(X), n \geq 1$, are decreasing and their diameters converge to zero. In fact, we have that

$$
\operatorname{diam}\left(S_{\left.\omega\right|_{n}}(X)\right)=s_{\omega_{1}} s_{\omega_{2}} \cdots s_{\omega_{n}} \operatorname{diam}(X) \leq s^{n} \operatorname{diam}(X) .
$$

Hence for an infinite word $\omega$, the set $\pi(\omega):=\bigcap_{n=1}^{\infty} S_{\left.\omega\right|_{n}}(X)$ is a singleton, and we can define a map $\pi: \mathbb{N}^{\infty} \rightarrow X$ which, in view of (2), is continuous. One obtains then the following limit set for the above infinite system of similarities:

$$
J:=\pi\left(\mathbb{N}^{\infty}\right)=\bigcup_{\omega \in \mathbb{N} \infty} \bigcap_{n=1}^{\infty} S_{\left.\omega\right|_{n}}(X) .
$$

This fractal limit set $J$ is not necessarily compact in the infinite case in contrast to that of the finite case (see [MaU], [MU1]). Let $\sigma: \mathbb{N}^{\infty} \rightarrow \mathbb{N}^{\infty}$ be the shift map on $\mathbb{N}^{\infty}$, that is, $\sigma(\omega)=\omega_{2} \omega_{3} \cdots$ where $\omega=\omega_{1} \omega_{2} \cdots$. Note that $\pi \circ \sigma(\omega)=S_{\omega_{1}}^{-1} \circ \pi(\omega)$, and hence, rewriting $\pi(\omega)=S_{\omega_{1}}(\pi(\sigma(\omega)))$, we see that $J$ satisfies the invariance condition:

$$
J=\bigcup_{i=1}^{\infty} S_{i}(J)
$$

One says that the above IFS satisfies the open set condition if there exists a bounded nonempty open set $U \subset X$ (in the topology of $X$ ), so that $S_{i}(U) \subset U$ for every $i \in \mathbb{N}$ and $S_{i}(U) \cap S_{j}(U)=\emptyset$ for every pair $i, j \in \mathbb{N}$ with $i \neq j$; and it satisfies the strong open set condition if $U$ can be chosen so that $U \cap J \neq \emptyset$ (see, e.g., $[\mathrm{H}],[\mathrm{M}])$. Since in our infinite case, the limit set $J$ may be noncompact, we will use a stronger condition; namely, we say that $\mathcal{S}$ is strongly separated if $\mathcal{S}$ satisfies the strong open set condition with a bounded open set $U$ and in addition $d\left(S_{i}(U), S_{j}(U)\right)>0$ for any $i \neq j$.

In the current paper, we assume that the infinite set of similarities satisfies the strongly separated condition. In the infinite systems case, the open set condition and the strong open set condition are not equivalent, unlike in the finite case (see $[\mathrm{SW}])$.

Let $\left(p_{1}, p_{2}, \ldots\right)$ be an infinite probability vector, with $p_{j}>0$ for all $j \geq 1$. Then there exists a unique Borel probability measure $\mu$ on $\mathbb{R}^{d}$ (see, e.g., $[\mathrm{H}]$, $[\mathrm{MaU}],[\mathrm{M}])$ such that

$$
\mu=\sum_{j=1}^{\infty} p_{j} \mu \circ S_{j}^{-1} .
$$

This measure $\mu$ is called the self-similar measure induced by the infinite IFS of self-similar mappings $\left(S_{j}\right)_{j \geq 1}$ and by the infinite probability vector $\left(p_{1}, p_{2}, \ldots\right)$, and is obtained as the projection $\pi_{*}\left(\nu_{\left(p_{1}, p_{2}, \ldots\right)}\right)$, where $\nu_{\left(p_{1}, p_{2}, \ldots\right)}$ is the product measure on $\mathbb{N}^{\infty}$ induced by $\left(p_{1}, p_{2}, \ldots\right)$. One defines the boundary at infinity $\mathcal{S}(\infty)$ as the set of accumulation points of sequences of type $\left(S_{i_{j}}\left(x_{i_{j}}\right)\right)_{j}$, for 
distinct integers $i_{j}$ (see [MaU]). The self-similar measure $\mu$ is supported in the closure $\bar{J}$ of the limit set $J$, which is given by $\bar{J}=J \cup \bigcup_{\omega \in \mathbb{N} \text { fin }} S_{\omega}(\mathcal{S}(\infty))$.

For the above fixed probability vector $\left(p_{1}, p_{2}, \ldots\right)$ and contraction vector $\left(s_{j}\right)_{j \geq 1}$ and for arbitrary $q, t \in \mathbb{R}$, we define the pressure function

$$
P(q, t)=\log \sum_{j=1}^{\infty} p_{j}^{q} s_{j}^{t} .
$$

Assume moreover that, for every $q \in[0,1]$, there exists a $u \in \mathbb{R}$ such that

$$
0 \leq P(q, u)<\infty .
$$

In this case, for an arbitrary $q \in \mathbb{R}$, let $\theta(q)=\inf \left\{t \in \mathbb{R}: \sum_{j=1}^{\infty} p_{j}^{q} s_{j}^{t}<\infty\right\}$. Then, for $q \in \mathbb{R}$ and $t \in(\theta(q), \infty)$, we have $P(q, t)<\infty$. This is similar to the condition of finiteness of entropy in the case of endomorphisms of Lebesgue spaces.

A particular case when the pressure is finite is when the infinite probability vector $\left(p_{1}, p_{2}, \ldots\right)$ and the contraction ratios $\left(s_{j}\right)_{j \geq 1}$ satisfy the following condition: there exists a constant $a>0$ such that $\sup _{j}\left|\log p_{j}-a \log s_{j}\right|<\infty$. Then there exists a constant $K \geq 1$ such that, for $j \geq 1$,

$$
K^{-1} s_{j}^{a} \leq p_{j} \leq K s_{j}^{a} .
$$

Condition (4) is then satisfied if we have (5), since we know that $s_{j}^{a} \leq K p_{j}, j \geq 1$, and since $\left(p_{1}, p_{2}, \ldots\right)$ is a probability vector; hence, for every $q \in[0,1]$ there exists some $t \in \mathbb{R}$ such that $\sum_{j=1}^{\infty} p_{j}^{q} s_{j}^{t}<\infty$.

The following lemmas are easy to prove.

\section{LEMMA 1.1}

Assuming that condition (4) is satisfied above, it follows that if $q \in \mathbb{R}$ is fixed, then the function $t \mapsto P(q, t)$ is strictly decreasing, convex, and continuous on $(\theta(q), \infty)$.

\section{LEMMA 1.2}

Assume that condition (4) is satisfied. Then for any $q \in[0,1]$, there exists a unique $t=\beta(q) \in(\theta(q), \infty)$ such that $P(q, \beta(q))=0$.

\section{Proof}

By Lemma 1.1, for a given $q \in[0,1]$, the function $P(q, t)$ is strictly decreasing and continuous on $(\theta(q), \infty)$. Since $0<P(q, u)<\infty$ for some $u \in(\theta(q), \infty)$, in order to conclude the proof it therefore suffices to show that $\lim _{t \rightarrow \infty} P(q, t)=-\infty$. For $t>u$,

$$
\begin{aligned}
P(q, t) & =\log \sum_{j=1}^{\infty} p_{j}^{q} s_{j}^{t}=\log \sum_{j=1}^{\infty} p_{j}^{q} s_{j}^{u} s_{j}^{t-u} \leq \log \sum_{j=1}^{\infty} p_{j}^{q} s_{j}^{u} s^{t-u} \\
& =P(q, u)+(t-u) \log s .
\end{aligned}
$$

Since $s<1$, it follows that $\lim _{t \rightarrow \infty} P(q, t)=-\infty$, and thus the lemma is obtained. 
LEMMA 1.3

The function $q \mapsto \beta(q)$ given in Lemma 1.2 is strictly decreasing, convex, and continuous on $[0,1]$.

Proof

Let $p=\sup \left\{p_{1}, p_{2}, \ldots\right\}$. Clearly, $p<1$. For any two points $q, q+\delta \in[0,1]$, where $\delta>0$, we have to show that $\beta(q+\delta)<\beta(q)$. If not, then let $\beta(q+\delta) \geq \beta(q)$. Then

$$
\begin{aligned}
0 & =P(q+\delta, \beta(q+\delta)) \leq P(q+\delta, \beta(q)) \\
& =\log \sum_{j=1}^{\infty} p_{j}^{q+\delta} s_{j}^{\beta(q)} \leq \log \sum_{j=1}^{\infty} p_{j}^{q} p^{\delta} s_{j}^{\beta(q)} ;
\end{aligned}
$$

hence, $0 \leq P(q, \beta(q))+\delta \log p=\delta \log p<0$, which is a contradiction. Thus $\beta(q+$ $\delta)<\beta(q)$. To show that $\beta(q)$ is convex, let $q_{1}, q_{2} \in[0,1]$ and $a_{1}, a_{2}>0$ with $a_{1}+a_{2}=1$. If $\beta(\cdot)$ is not convex, then there exist $a_{1}, a_{2}, q_{1}, q_{2}$ such that $\beta\left(a_{1} q_{1}+\right.$ $\left.a_{2} q_{2}\right)>a_{1} \beta\left(q_{1}\right)+a_{2} \beta\left(q_{2}\right)$. Then using Hölder's inequality, we have that

$$
\begin{aligned}
0 & =P\left(a_{1} q_{1}+a_{2} q_{2}, \beta\left(a_{1} q_{1}+a_{2} q_{2}\right)\right) \\
& <P\left(a_{1} q_{1}+a_{2} q_{2}, a_{1} \beta\left(q_{1}\right)+a_{2} \beta\left(q_{2}\right)\right) \\
& =\log \sum_{j=1}^{\infty} p_{j}^{a_{1} q_{1}+a_{2} q_{2}} s_{j}^{a_{1} \beta\left(q_{1}\right)+a_{2} \beta\left(q_{2}\right)} \\
& \leq \log \left(\sum_{j=1}^{\infty} p_{j}^{q_{1}} s_{j}^{\beta\left(q_{1}\right)}\right)^{a_{1}}\left(\sum_{j=1}^{\infty} p_{j}^{q_{2}} s_{j}^{\beta\left(q_{2}\right)}\right)^{a_{2}} \\
& =a_{1} P\left(q_{1}, \beta\left(q_{1}\right)\right)+a_{2} P\left(q_{2}, \beta\left(q_{2}\right)\right)=0,
\end{aligned}
$$

a contradiction. So $\beta\left(a_{1} q_{1}+a_{2} q_{2}\right) \leq a_{1} \beta\left(q_{1}\right)+a_{2} \beta\left(q_{2}\right)$, that is, $\beta(q)$ is convex and hence continuous.

The function $(q, t) \mapsto P(q, t)$ is called the topological pressure function corresponding to the given infinite IFS. The function $\beta(q)$, sometimes denoted by $T(q)$, is called the temperature function (as in $[\mathrm{HJK}+]$ ).

\section{REMARK 1.4}

If $q=0$, then from (3) we have that $\sum_{j=1}^{\infty} s_{j}^{\beta(0)}=1$, that is, $\beta(0)$ gives the Hausdorff $\operatorname{dimension} \operatorname{dim}_{\mathrm{H}}(J)$ of the infinite self-similar set $J$. (It was shown in [M] that this is the case.) Moreover, $P(1,0)=0$, which gives $\beta(1)=0$.

\section{The quantization coefficients for self-similar measures in the case of infinite systems}

For arbitrary $r>0$, let us define the auxiliary function $h:(0,1] \rightarrow \mathbb{R}$ by $h(x):=$ $\beta(x) /(r x), x \in(0,1]$, where $\beta(\cdot)$ was defined in Section 1 , in terms of the pressure 
function $P(\cdot)$ of our infinite system. We know that $\beta(1)=0$ and $\beta(0)=\operatorname{dim}_{\mathrm{H}}(J)$, and so $h(1)=0$ and $\lim _{x \rightarrow 0+} h(x)=\infty$. Moreover, the function $h$ is continuous and strictly decreasing on $(0,1]$. Hence, there exists a unique $q_{r} \in(0,1)$ such that $h\left(q_{r}\right)=1$, that is, $\beta\left(q_{r}\right)=r q_{r}$; hence, $P\left(q_{r}, \beta\left(q_{r}\right)\right)=0$. We assume also condition (4). Then, from the above definitions and lemmas it follows that for every $r>0$ there exists a unique number $\kappa_{r} \in(0, \infty), \kappa_{r}=\beta\left(q_{r}\right) /\left(1-q_{r}\right)$, and thus we have the formula

$$
P\left(\frac{\kappa_{r}}{r+\kappa_{r}}, \frac{r \kappa_{r}}{r+\kappa_{r}}\right)=0
$$

We now give the main result about quantization coefficients of the self-similar measure $\mu$ in the infinite system case.

\section{THEOREM 2.1}

Consider an infinite IFS of contractive similarities $\mathcal{S}=\left(S_{1}, S_{2}, \ldots\right)$ which satisfies the strongly separated condition, and let $J$ be its possibly noncompact limit set. Consider the infinite vector $\left(s_{1}, s_{2}, \ldots\right)$ consisting of the contraction rates of $\mathcal{S}$, and consider also an infinite probability vector $\left(p_{1}, p_{2}, \ldots\right)$ such that condition (4) above is satisfied. Let us consider $\mu$ to be the self-similar probability measure associated to $\mathcal{S}$ and to $\left(p_{1}, p_{2}, \ldots\right)$. Denote by $P(q, t)$ the corresponding pressure function, denote by $\beta(q)$ the zero of the function $P(q, \cdot)$, and for $r>0$, let $\kappa_{r}=\beta\left(q_{r}\right) /\left(1-q_{r}\right)$.

Then, for any $r \in(0, \infty)$ and for any $\kappa<\kappa_{r}<\kappa^{\prime}$, the following estimates on the lower/upper quantization coefficients of order $r$ for the self-similar measure $\mu$ (supported on $\bar{J}$ ) are true:

$$
0<\liminf _{n \rightarrow \infty} n V_{n, r}(\mu)^{\kappa / r} \quad \text { and } \quad \limsup _{n \rightarrow \infty} n V_{n, r}(\mu)^{\kappa^{\prime} / r}=0 .
$$

\section{Proof}

We first want to show that for $\kappa<\kappa_{r}$, the lower quantization coefficient $\underline{\mathcal{Q C}}_{\kappa, r}(\mu)$ is positive; that is, that $\liminf _{n \rightarrow \infty} n V_{n, r}(\mu)^{\kappa / r}>0$, where $\mu$ is the self-similar measure associated to $\left(S_{j}\right)_{j}$ and to the probabilistic vector $\left(p_{j}\right)_{j \geq 1}$ and where $\kappa_{r}$ is the unique number satisfying the sum condition

$$
\sum_{j=1}^{\infty}\left(p_{j} s_{j}^{r}\right)^{\frac{\kappa_{r}}{r+\kappa_{r}}}=1 .
$$

Let $\tilde{\nu}$ be the self-similar probability measure corresponding to the infinite system $(S, \gamma)$ where $S=\left\{S_{1}, S_{2}, \ldots\right\}$ and $\gamma=\left(\gamma_{1}, \gamma_{2}, \ldots\right)$ is the probability vector with $\gamma_{j}=\left(p_{j} s_{j}^{r}\right)^{\frac{\kappa_{r}}{r+\kappa_{r}}}, j \geq 1$. This measure $\tilde{\nu}$ can be constructed as the image, through the canonical projection $\pi$, of the product measure $\nu_{\left(\gamma_{1}, \gamma_{2}, \ldots\right)}$ on $\mathbb{N}^{\infty}$ associated to the probability vector $\left(\gamma_{1}, \gamma_{2}, \ldots\right)$; so we have $\tilde{\nu}=\pi_{*}\left(\nu_{\left(\gamma_{1}, \gamma_{2}, \ldots\right)}\right)$.

Consider now $U$ to be an open set satisfying the strongly separated condition; that is, $U \cap J \neq \emptyset, S_{j}(U) \subset U$, and $d\left(S_{i}(U), S_{j}(U)\right)>0, i \neq j$. Then it is easy to show that there exists a finite sequence of integers $\xi$ such that $J_{\xi} \subset U$, where we denote by $J_{\zeta}:=S_{\zeta}(J)$ for an arbitrary finite sequence $\zeta$. Let us take then a finite 
sequence $\xi$ as above, and define the positive constant $\eta_{0}:=1-\frac{1}{2} \gamma_{\xi}$. Then, for every nonempty set $V \subset J$ which is open with respect to the induced topology on $J$, it can be proved as in [GL3] that there exists an integer $n \in \mathbb{N}$ and finite sequences $\left(\sigma^{(k)}\right)_{1 \leq k \leq n}$ in $\mathbb{N}^{\text {fin }} \backslash\{\emptyset\}$ such that the sets $J_{\sigma^{(1)}}, \ldots, J_{\sigma^{(n)}}$ are pairwise disjoint in $V$ and satisfy the following condition (saying basically that their union has large $\tilde{\nu}$-measure):

$$
\tilde{\nu}\left(V \backslash \bigcup_{k=1}^{n} J_{\sigma^{(k)}}\right) \leq \eta_{0} \cdot \tilde{\nu}(V) .
$$

Moreover, employing the last inequality, one can then show that there exists a sequence $\left(\sigma^{(i)}\right)_{i}$ in $\mathbb{N}^{\text {fin }} \backslash\{\emptyset\}$ such that the associated sets $J_{\sigma^{(i)}}, i \geq 1$, are pairwise disjoint and satisfy

$$
\sum_{i=1}^{\infty} \tilde{\nu}\left(J_{\sigma^{(i)}}\right)=1 .
$$

We are now ready to prove the lower bound of the quantization coefficients for $\mu$. Let $0<r<\infty$ be fixed, and let $\kappa_{r}$ be as in (6), and let an arbitrary $\kappa<\kappa_{r}$. Then, we want to show that $\liminf _{n \rightarrow \infty} n V_{n, r}(\mu)^{\kappa / r}>0$.

By the formula in (8) and from the mutual disjointness of the sets $J_{\sigma^{(i)}}, i \geq 1$, we have that

$$
1=\sum_{i=1}^{\infty} \tilde{\nu}\left(J_{\sigma^{(i)}}\right)=\sum_{i=1}^{\infty}\left(p_{\sigma^{(i)}} s_{\sigma^{(i)}}^{r}\right)^{\frac{\kappa_{r}}{r+\kappa_{r}}} .
$$

However $\frac{\kappa}{r+\kappa}<\frac{\kappa_{r}}{r+\kappa_{r}}$; hence, there exists an associated positive integer $m=m(\kappa)$, such that

$$
\sum_{i=1}^{m}\left(p_{\sigma^{(i)}} s_{\sigma^{(i)}}^{r}\right)^{\frac{\kappa}{r+\kappa}} \geq 1
$$

Now from [GL1] it follows that, for every $n \in \mathbb{N}$, there exists an optimal set $Z_{n} \subset \mathbb{R}^{d}$ with $\operatorname{Card}\left(Z_{n}\right) \leq n$ and

$$
e_{n, r}^{r}(\mu)=\int_{J} d\left(x, Z_{n}\right)^{r} d \mu(x) .
$$

Let us define now $\delta_{n}=\sup _{x \in J} d\left(x, Z_{n}\right)$. Then one has $\lim _{n \rightarrow \infty} \delta_{n}=0$. But the sets $J_{\sigma^{(1)}}, \ldots, J_{\sigma^{(m)}}$ are pairwise disjoint, and moreover from the strongly separated condition $d\left(S_{i}(J), S_{j}(J)\right)>0$ for any $i \neq j$; therefore, we obtain the inequality

$$
\delta:=\min \left\{d\left(J_{\sigma^{(i)}}, J_{\sigma^{(j)}}\right): 1 \leq i, j \leq m, i \neq j\right\}>0,
$$

and from the above, $\delta=\delta(m)$ is independent of $s$. Thus, there must exist an integer $n_{0} \in \mathbb{N}$, such that $\delta_{n}<\frac{\delta}{2}$, for all $n \geq n_{0}$.

For $n \geq n_{0}$ and $i \in\{1,2, \ldots, m\}$, define the set $Z_{n, i}=\left\{a \in Z_{n}: d\left(a, J_{\sigma^{(i)}}\right) \leq\right.$ $\left.\delta_{n}\right\}$, and denote $k_{i}(n)=\operatorname{Card}\left(Z_{n, i}\right)$. By definition, $k_{i}(n) \geq 1$. Since the $Z_{n, i}$ 's $(i=1,2, \ldots, m)$ are mutually disjoint and contained in $Z_{n}$, we get moreover that $\sum_{i=1}^{m} k_{i}(n) \leq n$; hence, $k_{i}(n) \leq n-1(i=1,2, \ldots, m)$. Also as in [GL3] we obtain 
that

$$
\begin{aligned}
e_{n, r}^{r} & =\int d\left(x, Z_{n}\right)^{r} d \mu(x) \geq \sum_{i=1}^{m} \int_{J_{\sigma^{(i)}}} d\left(x, Z_{n}\right)^{r} d \mu(x) \\
& =\sum_{i=1}^{m} \int_{J_{\sigma^{(i)}}} d\left(x, Z_{n, i}\right)^{r} d \mu(x) \\
& =\sum_{i=1}^{m} p_{\sigma^{(i)}} s_{\sigma^{(i)}}^{r} \int_{J} d\left(x, S_{\sigma^{(i)}}^{-1}\left(Z_{n, i}\right)\right)^{r} d \mu(x) \geq \sum_{i=1}^{m} p_{\sigma^{(i)}} s_{\sigma^{(i)}}^{r} e_{k_{i}(n), r}^{r} .
\end{aligned}
$$

Define now $\chi=\chi(\kappa)=\min \left\{n e_{n, r}^{\kappa}: n \leq n_{0}\right\}$; then $\chi>0$. We show by induction that $\chi \leq n e_{n, r}^{\kappa}$ for $n \geq n_{0}$. In the induction step, let us assume that $\chi \leq j e_{j, r}^{\kappa}$ for $j \leq n-1$ and $n-1 \geq n_{0}$. Since $k_{i}(n) \leq n-1$, we can apply the induction step in the last displayed inequality, thus:

$$
e_{n, r}^{r} \geq \sum_{i=1}^{m} p_{\sigma^{(i)}} s_{\sigma^{(i)}}^{r} \chi^{\frac{r}{\kappa}} k_{i}(n)^{-\frac{r}{\kappa}} .
$$

Now, by the generalized Hölder's inequality, we have

$$
\sum_{i=1}^{m} p_{\sigma^{(i)}} s_{\sigma^{(i)}}^{r} k_{i}(n)^{-\frac{r}{\kappa}} \geq\left(\sum_{i=1}^{m}\left(p_{\sigma^{(i)}} s_{\sigma^{(i)}}^{r}\right)^{\frac{\kappa}{\kappa+r}}\right)^{1+\frac{r}{\kappa}} \cdot\left(\sum_{i=1}^{m} k_{i}(n)\right)^{-\frac{r}{\kappa}} .
$$

Recall however that $\sum_{i=1}^{m}\left(p_{\sigma^{(i)}} s_{\sigma^{(i)}}^{r}\right)^{\frac{\kappa}{r+\kappa}} \geq 1$ and $\sum_{i=1}^{m} k_{i}(n) \leq n$, hence $e_{n, r}^{r} \geq$ $\chi^{\frac{r}{\kappa}} n^{-\frac{r}{\kappa}}$, and $n e_{n, r}^{\kappa} \geq \chi$. Then by induction, for all $n \geq n_{0}, n e_{n, r}^{\kappa} \geq \chi>0$. Hence we obtain:

$$
\liminf _{n \rightarrow \infty} n e_{n, r}^{\kappa} \geq \chi(\kappa)>0,
$$

and therefore for arbitrary $\kappa<\kappa_{r}$, the $\kappa$-lower quantization coefficient of order $r$ for $\mu$ is positive.

We prove now the upper bound of the upper quantization coefficients $\overline{\mathcal{Q C}}_{r, \kappa^{\prime}}(\mu)$ in the infinite self-similar case, where $\kappa^{\prime}>\kappa_{r}$ is arbitrary.

Let us first fix a number $\kappa>\kappa_{r}$, and denote by $\eta:=\kappa /(r+\kappa)$. Then by the definition of $\kappa_{r}$, we have $\sum_{i \geq 1}\left(p_{i} s_{i}^{r}\right)^{\frac{\kappa_{r}}{r+\kappa_{r}}}=1$. So since $\eta>\kappa_{r} /\left(r+\kappa_{r}\right)$, there exists some number $\alpha=\alpha(\eta)$ such that

$$
\sum_{i \geq 1}\left(p_{i} s_{i}^{r}\right)^{\eta}<\alpha<1 .
$$

Notice now that, since $\bar{J}$ is compact, we can find a finite number of contractive similarities $T_{1}, \ldots, T_{K}$ on $X$ such that $S_{i}(X) \subset T_{1}(X) \cup \cdots \cup T_{K}(X), i \geq 1$. Without loss of generality we can assume that all sets $S_{j}(X)$ are contained in $T_{1}(X)$ for all $j \geq j_{0}$, for some large fixed integer $j_{0}$. Since $\alpha=\alpha(\eta)<1$, there exists some integer $N \geq j_{0}$ such that

$$
\left(\sum_{j>N} p_{j}\right)^{\eta}<\frac{1-\alpha}{2}
$$

As $\alpha$ depends on $\eta$, the above integer $N=N(\eta)$ also depends on $\eta$. Let us define now the finite system of contractive similarities $\tilde{S}_{i}, 1 \leq i \leq N+1$, where 
$\tilde{S}_{i}=S_{i}, 1 \leq i \leq N$, and $\tilde{S}_{N+1}=T_{1}$, under the above assumption about $T_{1}$. And define $\tilde{p}_{i}=p_{i}, 1 \leq i \leq N, \tilde{p}_{N+1}=\sum_{i>N} p_{i}$. We shall denote by $\tilde{s}_{i}$ the contraction ratio of $\tilde{S}_{i}$, for $1 \leq i \leq N+1$. Recall that, by our assumption, we have $S_{i}(X) \subset$ $\tilde{S}_{N+1}(X), \forall i>N$. On the other hand, from the self-similarity condition of the measure $\mu$, we have the decomposition

$$
\mu=\sum_{i \geq 1} p_{i} \mu \circ S_{i}^{-1}=\sum_{i=1}^{N} p_{i} \mu \circ S_{i}^{-1}+\sum_{j>N} p_{j} \mu \circ S_{j}^{-1} .
$$

For $\eta$ and $N$ as above, let us introduce also the following numbers from $(0,1)$ :

$$
\gamma_{i}:=\left(\tilde{p}_{i} \tilde{s}_{i}^{r}\right)^{\eta}, \quad 1 \leq i \leq N+1 .
$$

For a finite set $\mathcal{F}$ of integers, denote by $\mathcal{F}^{*}$ the set of all finite sequences of any length, with elements in $\mathcal{F}$. For a finite sequence $\omega=\left(\omega_{1}, \ldots, \omega_{p}\right) \in\{1, \ldots, N+$ $1\}^{*}, p \geq 1$, denote by $\gamma_{\omega}:=\gamma_{\omega_{1}} \cdots \gamma_{\omega_{p}}$. Also we denote by $\omega^{-}=\left(\omega_{1}, \ldots, \omega_{p-1}\right)$ the truncation of $\omega$ obtained by cutting the last element.

We now want to decompose $\mu$ successively, using (11) up to certain maximal finite sequences $\omega \in\{1, \ldots, N+1\}^{*}$, until we achieve that all the corresponding $\gamma_{\omega}$ 's are "almost equal" to $\frac{1}{n}$. Let us define then the following set of finite sequences determined by $N$ and $n$ :

$$
F_{n}:=\left\{\omega \in\{1, \ldots, N+1\}^{*}, \gamma_{\omega} \leq \frac{1}{n} \cdot \rho(N)^{-1}, \gamma_{\omega^{-}}>\frac{1}{n} \rho(N)^{-1}\right\},
$$

where $\rho(N):=\inf \left\{\gamma_{1}, \ldots, \gamma_{N+1}\right\}$. It follows that if $\omega \in F_{n}$, then $\gamma_{\omega}>\frac{1}{n}$. Also since we assumed that $\tilde{p}_{N+1}^{\eta}<(1-\alpha) / 2$ and $\sum_{i=1}^{N} \gamma_{i}<\alpha$, and recalling the definition of the $\gamma_{i}$ 's, we obtain that

$$
\sum_{i=1}^{N+1} \gamma_{i}<1
$$

Then, recalling that $\gamma_{\omega}>\frac{1}{n}, \omega \in F_{n}$, and since we have $1>\sum_{\omega \in F_{n}} \gamma_{\omega} \geq$ $\operatorname{Card}\left(F_{n}\right) \cdot \frac{1}{n}$, we obtain that

$$
\operatorname{Card}\left(F_{n}\right) \leq n
$$

In the identity (11) for $\mu$, we can then continue decomposing successively until reaching the value $\frac{1}{n}$ for $\gamma_{\omega}$, that is, we can split $\mu$ according to all finite sequences $\omega \in F_{n}$. In order to see this, let us deduce from (11) the following decomposition:

$$
\begin{aligned}
\mu= & \sum_{i=1}^{N} p_{i} \cdot\left(\sum_{j=1}^{N} p_{j} \mu \circ S_{j}^{-1}\right) \circ S_{i}^{-1}+\sum_{i=1}^{N} p_{i} \cdot\left(\sum_{j>N} p_{j} \mu \circ S_{j}^{-1}\right) \circ S_{i}^{-1} \\
& +\sum_{j>N} p_{j} \cdot\left(\sum_{k=1}^{N} p_{k} \mu \circ S_{k}^{-1}\right) \circ S_{j}^{-1}+\sum_{j>N} p_{j} \cdot\left(\sum_{k>N} p_{k} \mu \circ S_{k}^{-1}\right) \circ S_{j}^{-1} .
\end{aligned}
$$


Notice that if a set $B$ has a point in $S_{i} \tilde{S}_{j}(X)$ for some $i, j \in\{1, \ldots, N\}$, then we have

$$
\int d(x, B)^{r} d\left(\mu \circ S_{j}^{-1} \circ S_{i}^{-1}\right) \leq s_{i}^{r} s_{j}^{r} C,
$$

for a constant $C>0$. And if $B$ has a point in $S_{i} S_{j}(X)$ for some $1 \leq i \leq N$ and $j>N$, then

$$
\int d(x, B)^{r} d\left(\mu \circ S_{j}^{-1} \circ S_{i}^{-1}\right) \leq s_{i}^{r} \tilde{s}_{N+1}^{r} C,
$$

since $S_{j}(X) \subset \tilde{S}_{N+1}(X)$. If we take a set $B$ with at least $(N+1)^{2}$ points such that $B$ has a point in each of the sets $\tilde{S}_{i} \tilde{S}_{j}, i, j \in\{1, \ldots, N+1\}$, then, since $S_{i}(X) \subset \tilde{S}_{N+1}(X), i>N$, we obtain the following estimate for the $n$th quantization error of order $r$ of $\mu$ :

$$
\begin{aligned}
V_{n, r}(\mu) \leq & C \cdot\left(\sum_{i, j=1}^{N} p_{i} p_{j} s_{i}^{r} s_{j}^{r}+\sum_{i=1}^{N} p_{i} s_{i}^{r}\left(\sum_{j>N} p_{j}\right) \tilde{s}_{N+1}^{r}\right. \\
& \left.+\sum_{j=1}^{N} p_{j} s_{j}^{r}\left(\sum_{i>N} p_{i}\right) \tilde{s}_{N+1}^{r}+\sum_{j, k>N} p_{j} p_{k} \tilde{s}_{N+1}^{2 r}\right),
\end{aligned}
$$

where $C$ is a positive constant independent of $N$. Similarly we can do this argument for the set $F_{n}$ instead of $\{1, \ldots, N+1\}$, and we can take a set $B$ of cardinality $n$ which has points in each of the sets $\tilde{S}_{\omega}(X)$ for $\omega \in F_{n}$; this is possible since, as we saw in $(13), \operatorname{Card}\left(F_{n}\right) \leq n$. It similarly follows as above that

$$
\begin{aligned}
V_{n, r}(\mu) & \leq C \cdot \sum_{\omega \in F_{n}} \tilde{p}_{\omega} \tilde{s}_{\omega}^{r} \\
& =C \cdot\left(\frac{1}{n}\right)^{\frac{1-\eta}{\eta}} \rho(N)^{\frac{1-\eta}{\eta}} \cdot \sum_{\omega \in F_{n}} \gamma_{\omega} \leq C \cdot\left(\frac{\rho(N)}{n}\right)^{\frac{1-\eta}{\eta}}
\end{aligned}
$$

Recalling that $N$ depends on $\eta$ (and hence on $\kappa$ ), we obtain the following estimate for the $\kappa$-dimensional upper quantization coefficient of order $r$ of $\mu$ :

$$
\limsup _{n \rightarrow \infty} n V_{n, r}(\mu)^{\kappa / r} \leq C(\kappa)<\infty,
$$

where $C(\kappa)$ is a positive constant depending on $\kappa$. In fact if we now take $\kappa^{\prime}$ arbitrarily larger than $\kappa$ and since $\lim _{n \rightarrow \infty} V_{n, r}(\mu)=0$, then we see that

$$
\limsup _{n \rightarrow \infty} n V_{n, r}(\mu)^{\kappa^{\prime} / r}=0 \text {. }
$$

From the above inequalities (9) and (14) we obtain also computable estimates for the lower and the upper quantization coefficients of order $r$ for the probability measure $\mu$. We do not know if the $\kappa_{r}$-dimensional upper quantization coefficient for $\mu$ of order $r$ is always finite in the case of infinite systems. In particular, from the estimates above for lower/upper quantization coefficients for $\mu$ and by taking 
$\kappa, \kappa^{\prime} \rightarrow \kappa_{r}$, we obtain that the quantization dimension of order $r$ of $\mu$ exists, and it is equal to $\kappa_{r}$.

\section{COROLLARY 2.2}

In the setting of Theorem 2.1, it follows that the quantization dimension $D_{r}(\mu)$ exists and $D_{r}(\mu)=\kappa_{r}$.

\section{REMARK 2.3}

We notice that, in order to obtain the proof of Theorem 2.1 and of Corollary 2.2, it is not possible to use finite truncations with $M$ elements $\mathcal{S}_{M}$ of the system and associated self-similar measures $\mu_{M}$, and then to consider $\log V_{n_{k}, r}\left(\mu_{M}\right)$ when $n_{k} \rightarrow \infty$, followed by the use of the estimates for the quantization dimension of $\mu_{M}$ from the finite case. This problem is due to the fact that the speed of convergence in $n_{k}$, in the formula for the quantization dimension of $\mu_{M}$, actually depends on each $M$ (when $M \rightarrow \infty$ ).

We give now some infinite systems where one can say more about the quantization process.

Examples. Consider a sequence of numbers $\left(s_{i}\right)_{i \geq 1}$ in the interval $(0,1)$ such that $s_{i}=\gamma^{i}, i \geq 1$, for some $\gamma \in(0,1 / 2)$. Let us also take $p_{i}=s_{i}^{a}=\gamma^{a i}, i \geq 1$, and $p=\left(p_{1}, p_{2}, \ldots\right)$; in order to make $p$ a probabilistic vector, we will choose $a=\log 2 /|\log \gamma|$.

We then take the strongly separated infinite IFS $\mathcal{S}$, formed by the sequence of similarities $\mathcal{S}=\left(S_{i}\right)_{i \geq 1}$ of the unit disk $\Delta(0,1)$ having contraction rates $s_{i}$, respectively, and such that the boundary at infinity $\mathcal{S}(\infty)$ is equal to the unit circle $S^{1}$. Consider also the self-similar probability measure $\mu$, associated to $\mathcal{S}$ and $p$. Then, the self-similar measure $\mu$ is supported on the closure $\bar{J}$, which in this case is given by

$$
\bar{J}=J \cup \bigcup_{\omega \in \mathbb{N}_{\text {fin }}} S_{\omega}(\mathcal{S}(\infty))=J \cup \bigcup_{\omega \in \mathbb{N}^{\text {fin }}} S_{\omega}\left(S^{1}\right) .
$$

We notice that in this case $H D(J)<1$, but the lower box dimension of $J$ is larger than or equal to 1, since $\overline{\operatorname{dim}}_{B}(J) \geq \overline{\operatorname{dim}}_{B}(\mathcal{S}(\infty))=1$. Now, one wants to estimate the quantization coefficients for the measure $\mu$. According to Theorem 2.1, the quantization dimension of $\mu$ is equal to $\kappa_{r}$, where $\kappa_{r}$ satisfies

$$
\sum_{i \geq 1}\left(p_{i} s_{i}^{r}\right)^{\frac{\kappa_{r}}{r+\kappa_{r}}}=1
$$

In our case, the above sum is just the sum for a geometric series; hence, we obtain with the above expression for $s_{i}, p_{i}$, and the above exponent $a$ that

$$
\sum_{i \geq 1}\left(\gamma^{(a+r) t}\right)^{i}=1
$$


where $t=\kappa_{r} /\left(r+\kappa_{r}\right)$. Hence $t=\log 2 /((a+r)|\log \gamma|)=\kappa_{r} /\left(r+\kappa_{r}\right)$. Therefore, we obtain the quantization dimension

$$
D_{r}(\mu)=\kappa_{r}=\frac{r \log 2}{(a+r)|\log \gamma|-\log 2}=\frac{\log 2}{|\log \gamma|} .
$$

It is interesting to note that, in this particular case, the quantization dimension $D_{r}(\mu)$ does not depend on $r$. In general, however, if the $p_{j}$ 's are not of the form above, then the quantization dimension $D_{r}(\mu)$ should depend on $r$. We have also from Theorem 2.1 that the lower/upper quantization coefficients for $\mu$ satisfy:

$$
\begin{gathered}
0<\liminf _{n \rightarrow \infty} n V_{n, r}(\mu)^{\frac{\kappa}{r}} \quad \text { and } \\
\limsup _{n \rightarrow \infty} n V_{n, r}(\mu)^{\frac{\kappa^{\prime}}{r}}=0, \quad \forall \kappa<\log 2 /|\log \gamma|<\kappa^{\prime} .
\end{gathered}
$$

We notice that this example can be modified so that the images $S_{i}(\Delta)$ are arranged differently inside $\Delta$, and that the boundary at infinity $\mathcal{S}(\infty)$ is more complicated, for instance, we can imagine an example where it is a countable union of concentric circles $C_{n}, n \geq 1$, centered at 0 , with radii $c_{n}$ going to 0 . The corresponding self-similar measure $\mu$ will then be supported on the closure of the limit set $J$, namely, on the compact set

$$
\bar{J}=J \cup \bigcup_{\omega \in \mathbb{N}^{\text {fin }}} S_{\omega}\left(\bigcup_{n} C_{n} \cup\{0\}\right) .
$$

Still, if we keep the same contraction rates $s_{i}$ and the probability vector $p=$ $\left(p_{1}, p_{2}, \ldots\right)$ as before, then we will obtain the same quantization dimension $\kappa_{r}$ and quantization coefficient estimates as above.

We want now to approximate the self-similar measure $\mu$ with discrete measures of finite support. Denote by $\mathcal{M}$ the set of probability measures on the compact set $X \subset \mathbb{R}^{d}$. Then,

$$
d_{H}(\mu, \nu):=\sup \left\{\left|\int_{X} g d \mu-\int_{X} g d \nu\right|: \operatorname{Lip} g \leq 1\right\}, \quad(\mu, \nu) \in \mathcal{M} \times \mathcal{M},
$$

defines a metric on $\mathcal{M}$. Then $\left(\mathcal{M}, d_{H}\right)$ is a compact metric space (see [B]). It is known that the $d_{H}$-topology and the weak topology coincide on the space of probabilities with compact support (see [Mat]). In our case all measures are compactly supported.

First, since $X$ is compact we have $\int\|x\|^{r} d \mu(x)<\infty$, for any probability measure $\mu$ on $X$. For $r \in(0, \infty)$ and for two arbitrary probabilities $\mu_{1}, \mu_{2}$, the $L_{r}$-Kantorovich-Wasserstein metric is defined by the following formula (see, e.g., [GL1]):

$$
\rho_{r}\left(\mu_{1}, \mu_{2}\right)=\inf _{\nu}\left(\int\|x-y\|^{r} d \nu(x, y)\right)^{\frac{1}{r}}
$$

where the infimum is taken over all Borel probabilities $\nu$ on $X \times X$ with fixed marginal measures $\mu_{1}$ and $\mu_{2}$ such that $\left(\pi_{1}\right)_{*}(\nu)=\mu_{1}$ and $\left(\pi_{2}\right)_{*}(\nu)=\mu_{2}$ for the canonical projections $\pi_{1}, \pi_{2}$ on the first and second coordinates, respectively. 
Note that the weak topology, the topology induced by $d_{H}$, and the topology induced by the $L_{r}$-metric $\rho_{r}$ all coincide on the space $\mathcal{M}$ (see for example [Ru]). Let us notice also that, for $r=1$, the $\rho_{1}$-metric is in fact equal to the $d_{H}$-metric in the compact case, as shown by Kantorovich (see [GL1]).

The next lemma relates the quantization errors for a probability measure $P$ to the $L_{r}$-Kantorovich-Wasserstein distances between $P$ and discrete measures.

LEMMA 2.4 ([GL1, LEMMA 3.4])

Let $\mathcal{P}_{n}$ denote the set of all discrete probability measures $Q$ on $X$ with $|\operatorname{supp}(Q)| \leq n$. Then for any probability $P$, we have that

$$
V_{n, r}(P)=\inf _{Q \in \mathcal{P}_{n}} \rho_{r}^{r}(P, Q) .
$$

Now by using Lemma 2.4 and Theorem 2.1, we obtain the following result about the asymptotic behavior in $n$ of the approximations in the $L_{r}$-metric of $\mu$, with discrete measures supported on $n$ points, when $n$ increases to $\infty$.

\section{COROLLARY 2.5}

In the setting of Theorem 2.1, let us consider the associated self-similar probability measure $\mu$. Then, for every $r \in(0, \infty)$, there exists a unique number $\kappa_{r} \in(0, \infty)$ such that for arbitrary $\kappa, \kappa^{\prime}$ with $\kappa<\kappa_{r}<\kappa^{\prime}$, the $L_{r}$-approximations of $\mu$ with discrete measures on $n$ points behave asymptotically as:

$$
0<\liminf _{n \rightarrow \infty} n^{\frac{1}{\kappa}} \cdot \inf _{Q \in \mathcal{P}_{n}} \rho_{r}(\mu, Q), \quad \text { and } \quad \limsup _{n \rightarrow \infty} n^{\frac{1}{\kappa^{\prime}}} \cdot \inf _{Q \in \mathcal{P}_{n}} \rho_{r}(\mu, Q)=0 .
$$

\section{References}

[B] M. Barnsley, Fractals Everywhere, Academic Press, Boston, 1988. MR 0977274.

[F] K. J. Falconer, The multifractal spectrum of statistically self-similar measures, J. Theoret. Probab. 7 (1994), 681-702. MR 1284660. DOI 10.1007/BF02213576.

[GG] A. Gersho and R. M. Gray, Vector Quantization and Signal Compression, Kluwer Academic, Boston, 1992.

[GL1] S. Graf and H. Luschgy, Foundations of Quantization for Probability Distributions, Lecture Notes in Math. 1730, Springer, Berlin, 2000. MR 1764176. DOI 10.1007/BFb0103945.

[GL2] Asymptotics of the quantization errors for self-similar probabilities, Real Anal. Exchange 26 (2000/01), 795-810. MR 1844394.

[GL3] , The quantization dimension of self-similar probabilities, Math.

Nachr. 241 (2002), 103-109. MR 1912380.

DOI 10.1002/1522-2616(200207)241:1<103::AID-MANA103>3.0.CO;2-J. 
[H] J. E. Hutchinson, Fractals and self-similarity, Indiana Univ. Math. J. 30 (1981), 713-747. MR 0625600. DOI 10.1512/iumj.1981.30.30055.

[HJK+] T. C. Halsey, M. H. Jensen, L. P. Kadanoff, I. Procaccia, and B. I. Shraiman, Fractal measures and their singularities: The characterization of strange sets, Phys. Rev. A (3) 33 (1986), 1141-1151. MR 0823474. DOI 10.1103/PhysRevA.33.1141.

[LM] L. J. Lindsay and R. D. Mauldin, Quantization dimension for conformal iterated function systems, Nonlinearity 15 (2002), 189-199. MR 1877974. DOI 10.1088/0951-7715/15/1/309.

[Mat] P. Mattila, Geometry of Sets and Measures in Euclidean Spaces, Cambridge Stud. Adv. Math. 44, Cambridge Univ. Press, Cambridge, 1995. MR 1333890. DOI 10.1017/CBO9780511623813.

[MaU] R. D. Mauldin and M. Urbański, Dimensions and measures in infinite iterated function systems, Proc. London Math. Soc. (3) 73 (1996), 105-154. MR 1387085. DOI 10.1112/plms/s3-73.1.105.

[MU] E. Mihailescu and M. Urbański, Hausdorff dimension of the limit set of conformal iterated function systems with overlaps, Proc. Amer. Math. Soc. 139 (2011), 2767-2775. MR 2801617. DOI 10.1090/S0002-9939-2011-10704-4.

[MU1] _ _ "Hausdorff dimension of the limit set of countable conformal iterated function systems with overlaps" in Fractal Geometry and Dynamical Systems in Pure and Applied Mathematics, I: Fractals in Pure Mathematics, Contemp. Math. 600, Amer. Math. Soc., Providence, 2013, 273-289. MR 3203406. DOI 10.1090/conm/600/11950.

[MU2] - Random countable iterated function systems with overlaps and applications, preprint, arXiv:1405.2942v2 [math.DS].

[M] M. Moran, Hausdorff measure of infinitely generated self-similar sets, Monatsh. Math. 122 (1996), 387-399. MR 1418125. DOI 10.1007/BF01326037.

[R1] M. K. Roychowdhury, Lower quantization coefficient and the F-conformal measure, Colloq. Math. 122 (2011), 255-263. MR 2775174. DOI 10.4064/cm122-2-11.

[R2] Quantization dimension for infinite self-similar probabilities, J. Math. Anal. Appl. 383 (2011), 499-505. MR 2812400. DOI 10.1016/j.jmaa.2011.05.044.

[Ru] L. Rüschendorf, "Wasserstein metric" in Encyclopedia of Mathematics, Kluwer, Dordrecht, 2001.

[SW] T. Szarek and S. Wedrychowicz, The OSC does not imply the SOSC for infinite iterated function systems, Proc. Amer. Math. Soc. 133 (2004), 437-440. MR 2093065. DOI 10.1090/S0002-9939-04-07708-1.

[Za] P. L. Zador, Asymptotic quantization error of continuous signals and the quantization dimension, IEEE Trans. Inform. Theory 28 (1982), 139-149. MR 0651809. DOI 10.1109/TIT.1982.1056490. 
[Z] S. Zhu, The lower quantization coefficient of the F-conformal measure is positive, Nonlinear Anal. 69 (2008), 448-455. MR 2426261. DOI 10.1016/j.na.2007.05.031.

Mihailescu: Institute of Mathematics "Simion Stoilow" of the Romanian Academy, P.O. Box 1-764, RO 014700, Bucharest, Romania; Eugen.Mihailescu@imar.ro

Roychowdhury: Department of Mathematics, The University of Texas-Pan American, 1201 West University Drive, Edinburg, Texas 78539, USA; roychowdhurymk@utpa.edu 